\title{
Rampant historical mitochondrial genome introgression between two species of green pond frogs, Pelophylax nigromaculatus and P. plancyi
}

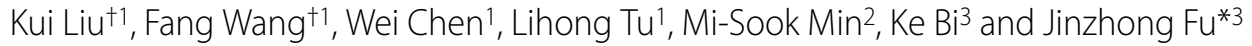

\begin{abstract}
Background: Mitochondrial introgression may result in the mitochondrial genome of one species being replaced by that of another species without leaving any trace of past hybridization in its nuclear genome. Such introgression can confuse the species genealogy estimates and lead to absurd inferences of species history. We used a phylogenetic approach to explore the potential mitochondrial genome introgression event(s) between two closely related green pond frog species, Pelophylax nigromaculatus and P. plancyi.

Results: DNA sequence data of one mitochondrial and two nuclear genes from an extensive sampling of the two species were collected, and the genealogies of the three genes were constructed and compared. While the two nuclear genes congruently showed mutual reciprocal monophyly of both species, the mitochondrial phylogeny separated a Korean P. nigromaculatus clade, a paraphyletic central China P. plancyi assemblage, and a large wellsupported introgression clade. Within the introgression clade, the mitochondrial haplotypes of the two species were mixed together. This reticulated pattern can be most parsimoniously explained by an ancient mitochondrial introgression event from P. plancyi to P. nigromaculatus that occurred at least 1.36 MYA, followed by multiple recent introgression events from P. nigromaculatus back to P. plancyi within the last $0.63 \mathrm{MY}$. The re-constitution of previously co-adapted genomes in $P$. plancyi may be responsible for the recent rampant introgression events. The Korean $P$. nigromaculatus clade likely represents the only surviving "true" mitochondrial lineage of $P$. nigromaculatus, and the central China P. plancyi assemblage likely represents the "original" P. plancyi mitochondrial lineage. Refugia in the Korean Peninsula and central China may have played a significant role in preserving these ancient lineages.

Conclusions: The majority of individuals in the two species have either introgressed (P. nigromaculatus) or reclaimed ( $P$. plancyi) mitochondrial genomes while no trace of past hybridization in their nuclear genomes was detected. Asymmetrical reproductive ability of hybrids and continuous backcrossing are likely responsible for the observed mitochondrial introgression. This case is unique in that it includes an ancient "forward" introgression and many recent "backward" introgressions, which re-constitutes the original nuclear and mitochondrial genomes of P. plancyi. This hybrid system provides an excellent opportunity to study cyto-nuclear interaction and co-adaptation.
\end{abstract}

\section{Background}

Historical mitochondrial introgression often results in the mitochondrial genome of one species being replaced by that of another species without leaving any trace of hybridization in its nuclear genome. The introgressed genome can become fixed in some populations and

* Correspondence: jfu@uoguelph.ca

3 Department of Integrative Biology, University of Guelph, Guelph, Ontario N1G 2W1, Canada

+ Contributed equally

Full list of author information is available at the end of the article extend to a large portion of the recipient species' distribution. Wilson and Bernatchez termed this phenomenon "ghost of hybrids past" [1]. For example, Melo-Ferreira et al. found mitochondrial introgression from the mountain hare (Lepus timidus) to three other hare species, L. granatensis, L. europaeus and L. castroviejoi in the Iberian Peninsula [2,3]. Although L. timidus is currently not sympatric with the three Iberian hares, distribution range expansion and retraction during glaciation might have created opportunities for the species to hybridize. Selective advantage of the L. timidus mitochondrial genome 
over others was attributed to the widespread presence of the introgressed mitochondrial genome. McGuire et al. also presented a case study in lizards of the genus Crotaphytus [4]. The mitochondrial genome of $C$. collaris has replaced that of $C$. reticulatus in approximately twothirds of its range via an ongoing selective sweep. A unidirectional mitochondrial introgression also took place from C. collaris to C. bicinctores. Introgressive hybridization may have occurred repeatedly but was temporally separated throughout at least the latter half of the Pleistocene. Other cases of mitochondrial introgression have been reported in diverse metazoan taxa including carabid beetles [5], fruit flies [6], brook charr [7], megophryid frogs [8], Sika deer [9], African elephants [10], and pocket gophers [11].

Such processes have a number of significant implications in biology. (1) Mitochondrial gene introgression can confuse the estimated genealogy of a species, because an introgressed genome will not reveal any history before the introgression events [12] and the mix of introgressed and original genomes within a species could lead to absurd inferences of the species history. Therefore, cautions should always be exercised when using mitochondrial DNA (mtDNA) alone to infer demography and evolutionary history of a species. This is important because in the last three decades, we have witnessed a great number of "phylogeographic" studies using mtDNA as a sole marker $[13,14]$. (2) Introgression often disrupts the co-evolution between the mitochondrial and nuclear genomes. Genes from both genomes encode several proteins that are critical to metabolism and an abrupt disassociation between the genomes may interfere not only their respective normal function but also the established genomic interactions and co-adaptation [15]. (3) Massive introgression events were often suggested to be adaptive processes driven by natural selection $[1,4,12]$. The spontaneous breakdown and reconstruction of mitochondrial-nuclear genome associations may open a unique window to examine the adaptation process of a newly introgressed mitochondrial genome, as well as to better understand the interactive dynamics and co-adaptive functioning between mitochondrial and nuclear genomes.

Kim et al. first suggested a possible mitochondrial genome introgression between two green pond frog species, Pelophylax nigromaculatus and P. plancyi chosenicus [16]. Using mitochondrial cytochrome $b$ partial sequences, they found that the Japanese P. nigromaculatus is more closely related to Korean P. plancyi chosenicus than to Korean $P$. nigromaculatus, while their nuclear gene data (allozymes) unequivocally group the $P$. nigromaculatus populations together. Subsequently, they hypothesized a possible mitochondrial genome introgression event between the two species. Nevertheless, the extent, timing and directions of introgression were unresolved because of their small sample size $(n=7)$. Green pond frogs in the genus Pelophylax (formerly part of the genus Rana) are no stranger to inter-specific hybridization. Pelophylax esculentus, a species of hybrid origin between $P$. lessonae and $P$. ridibunda, is probably the best studied case of inter-specific hybridization [17]. Both $P$. nigromaculatus and P. plancyi are common and widespread in eastern Asia, and their distribution ranges largely overlap (Figure 1). Ecologically, P. nigromaculatus is a generalist and occurs in a wide range of habitats including small to large ponds and rivers. On the other hand, $P$. plancyi requires more specific habitats and is most commonly seen in large well-vegetated ponds, particularly those with lotus plants. Both species are spring breeders and occasionally use the same breeding sites. The gross morphology of the two species is similar but they can be easily distinguished. While males of $P$. nigromaculatus have a pair of external vocal sacs, males of $P$. plancyi have a pair of internal ones or do not have vocal sacs at all. In addition, $P$. plancyi has a conspicuous dark line along the back of its thigh that is lacking in $P$. nigromaculatus. Several other names have been proposed for geographic populations of $P$. plancyi, including $P$. fukienensis, $P$. hubeiensis, and $P$. chosenicus. Some authors treat them as valid species [18] while others treat them as subspecies of P. plancyi ([19]; see Frost [20] for references and comments). In this study, we refer all populations allocated to these names as the P. plancyi complex to facilitate our presentation.

Following the lead of Kim et al.'s study [16], we use a phylogenetic approach to explore the potential mitochondrial genome introgression between $P$. nigromaculatus and $P$. plancyi, and to detail its extension in both time and space by extensive sampling of both species across their ranges. Recent development in DNA sequencing technology allows us to obtain large amounts of sequence data from both the mitochondrial and nuclear genomes. By comparing their gene genealogies, gene introgression events can be revealed. If there was no historical mitochondrial introgression between $P$. nigromaculatus and $P$. plancyi, both the nuclear and mitochondrial genes would group haplotypes from each of the two species respectively in the same fashion, and Kim et al.'s hypothesis [16] would be rejected. If introgressions occurred between the two species, a phylogeny based on nuclear genes would group haplotypes from each of the two species, but part or all of the mitochondrial haplotypes from one species would nest within the other species. 


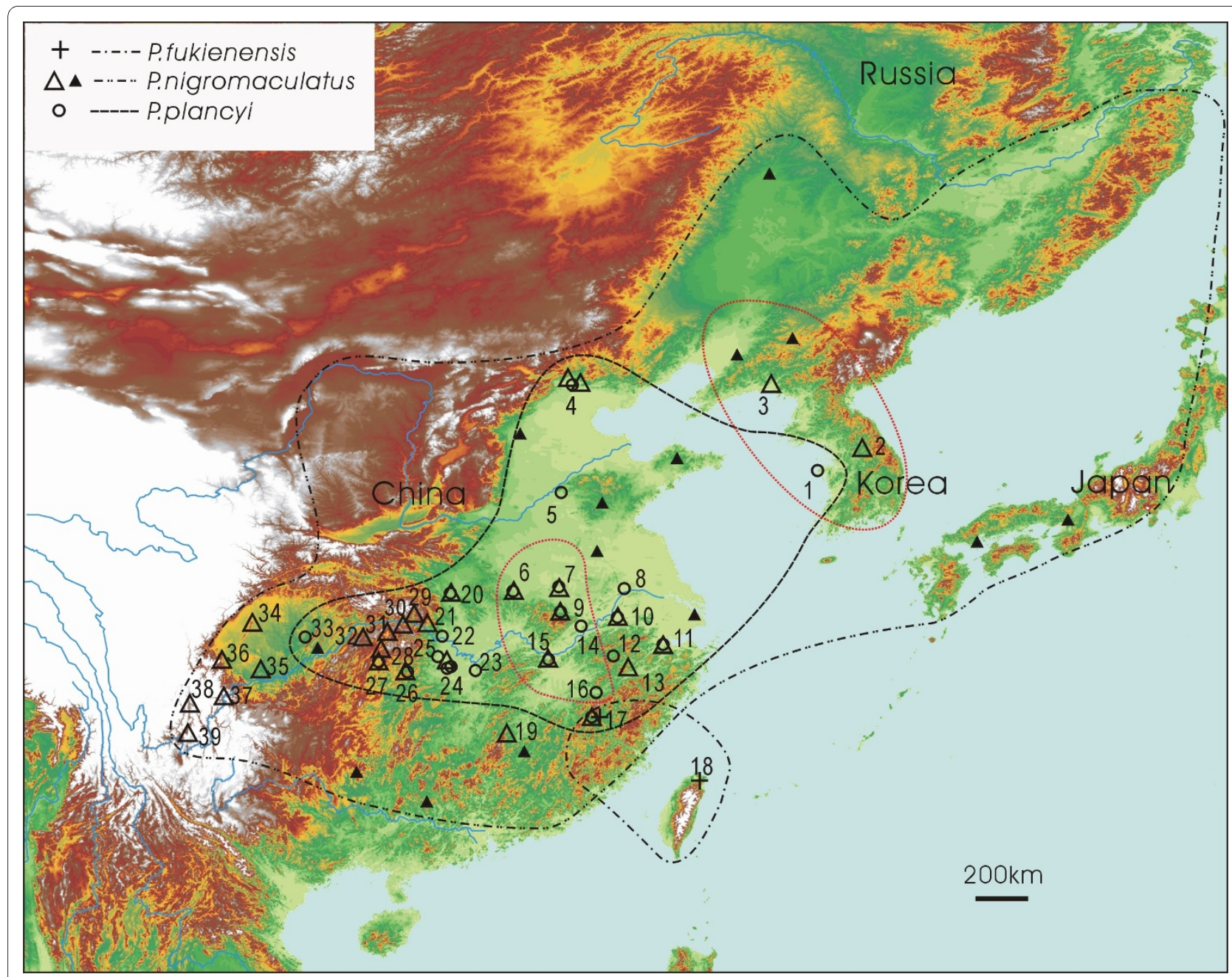

Figure 1 Species distribution map and sampling sites of Pelophylax fukienensis, P. nigromaculatus and P. plancyi. Cross $=$ Pelophylax fukienensis; Circle $=P$. plancyi; Triangle $=P$. nigromaculatus. Solid triangles represent samples from GenBank. Only selected sampling sites from GenBank are presented here to demonstrate geographic coverage. The red dotted lines outline the distribution of two ancient lineages of "original" mitochondrial genomes, which are possibly associated with the assumptive Korean refugium and the central China refugium during glaciations.

\section{Results}

\section{Nuclear proopiomelanocortin gene (POMC) and tyrosinase} gene (TYR) genealogies

Sequence data were collected from two nuclear genes (POMC and TYR). We first conducted a recombination test for the nuclear gene data and did not detect any recombination event at both global and pairwise levels. Therefore, all data were proceeded for phylogenetic analyses without modification. Both Bayesian inference and maximum parsimony methods were used to construct the gene genealogy.

A total of 66 POMC sequences from 59 samples for Pelophylax nigromaculatus (including one from GenBank) and 488 sequences from 321 samples for P. plancyi complex were gathered. Two sequences for two outgroup taxa, Lithobates catesbeianus and Staurois latopalmatus were gathered from GenBank. A total of 611 nucleotide sites were confidently resolved and 139 haplotypes were identified, including two for the outgroup taxa. Among the ingroup members, 83 sites were variable.

For the Bayesian analysis, the $\mathrm{HKY}+\mathrm{I}+\mathrm{G}$ model was selected as the best-fit model by the hierarchical likelihood ratio test. Figure 2 shows one of the Bayesian trees with posterior probabilities of the basal nodes mapped on the tree. Both the resolution of the Bayesian consensus tree and the posterior probabilities were low (Figure 2); this was not surprising given the small number of variable sites. However, three clades were clearly depicted on the tree with moderate to high nodal supports. A P. plancyi clade included all haplotypes from $P$. plancyi complex except $P$. fukienensis, and a $P$. nigromaculatus clade included all haplotypes of $P$. nigromaculatus except CNU5268. CNU5268 (site 15) was a heterozygote and its two haplotypes were grouped in the $P$. plancyi clade and 


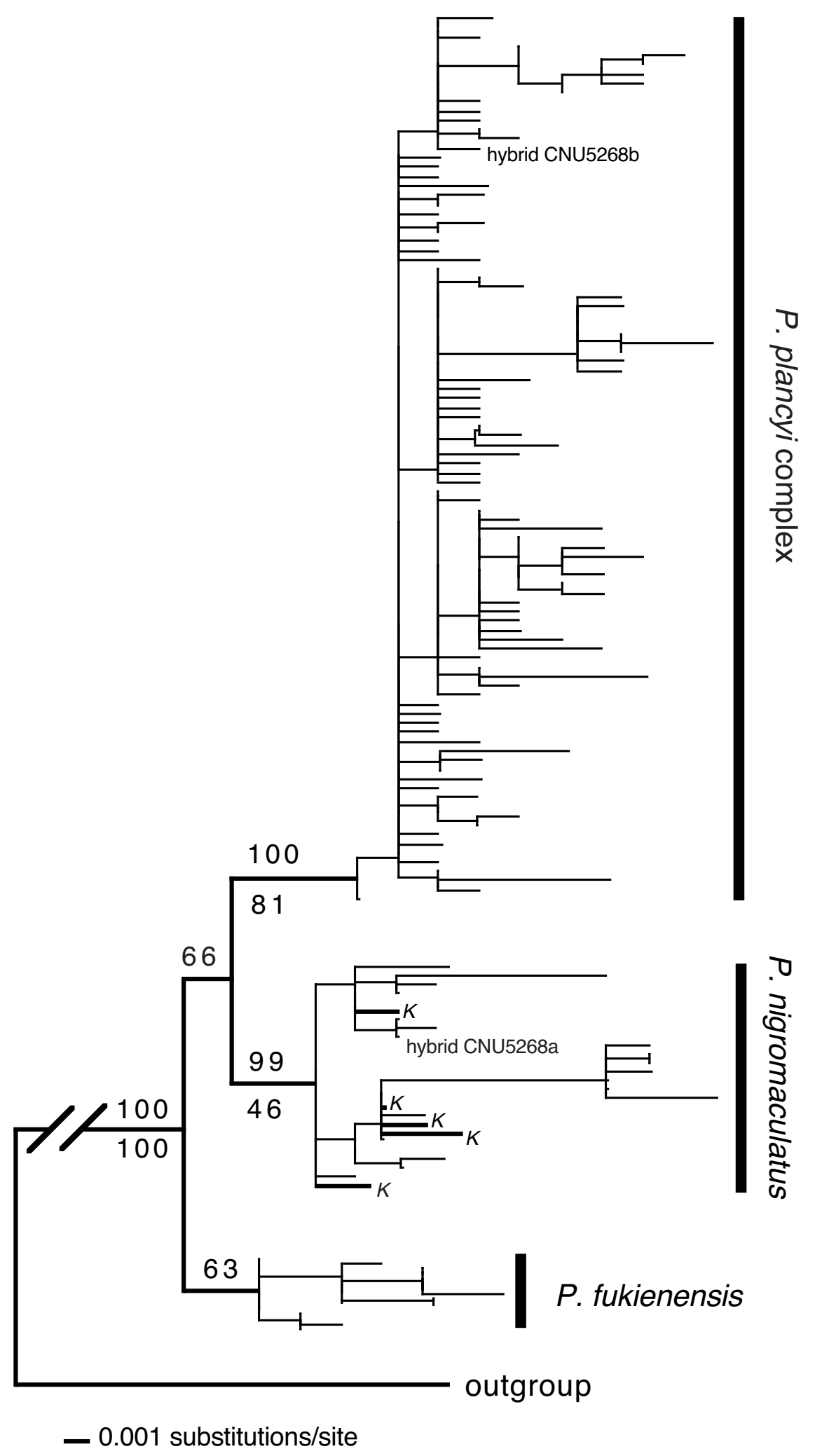

Figure 2 A Bayesian tree from the POMC gene data. The numbers above the branches are Bayesian posterior probabilities and the numbers below the branches are bootstrap proportions from the parsimony analysis. Bold lines and the " $K$ " indicate haplotypes from the Korean nigromaculatus clade 
the $P$. nigromaculatus clade, respectively. Both clades received high posterior probabilities (0.99 and 1.00). A third clade, the P. fukienensis clade, included all samples from $P$. fukienensis, with a low posterior probability (0.63). For the maximum parsimony analysis, 41 sites were phylogenetically informative. The parsimony analysis found more than 400,000 equally most parsimonious trees (MPTs) with 130 steps, a consistency index (CI) of 0.4538 and a retention index (RI) of 0.8483 . The strict consensus tree (tree not shown) was similar but less resolved compared to the Bayesian consensus tree. Again, a $P$. nigromaculatus clade and a $P$. plancyi clade were resolved with exactly the same membership as the Bayesian tree. The relationships within each clade were largely unresolved. However, the haplotypes of P. fukienensis samples did not form a monophyletic group. Rather, they formed a paraphyletic assemblage at the base of the tree.

A total of 77 TYR sequences from 60 samples for Pelophylax nigromaculatus (including three from GenBank) and 481 sequences from 321 samples for P. plancyi were gathered. Three sequences for three outgroup taxa, Babina pleuraden, Rana shuchinae, and Pelophylax lessonae, were gathered from GenBank. A total of 601 nucleotide sites were confidently resolved and 79 haplotypes were identified, including three for the outgroup. Among the ingroup members, 42 sites were variable and 23 were phylogenetically informative.

The parsimony analysis found better-resolved trees than did the Bayesian analysis, and therefore, a simplified

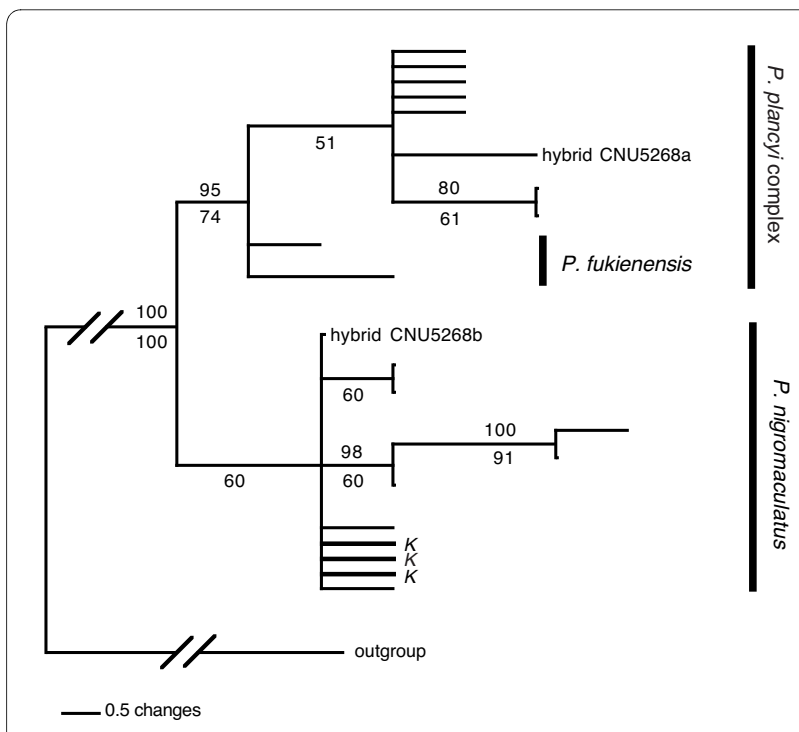

Figure 3 The simplified phylogram of the strict consensus tree from the parsimony analysis of the TYR gene data. The numbers above the branches are Bayesian posterior probabilities from the Bayesian analysis and the numbers below the branches are bootstrap proportions. Bold lines and the " $K$ " indicate haplotypes from the Korean nigromaculatus clade; all these haplotypes are shared between members of the Korean nigromaculatus clade and others. phylogram of the strict consensus tree from the parsimony analysis is presented in Figure 3. The analysis found more than 400,000 MPTs with 93 steps $(\mathrm{CI}=0.5161$; $\mathrm{RI}=$ 0.8193). Both the resolution of the tree and the bootstrap supports were low, which was not surprising given the small number of informative characters. However, two clades, a $P$. nigromaculatus clade and a P. plancyi clade (including $P$. fukienensis) were clearly depicted on the strict consensus tree with moderate nodal supports (Figure 3). For the Bayesian analysis, a SYM+I+G model was selected as the best-fit model by the hierarchical likelihood ratio test. Similar to the parsimony tree, the Bayesian tree also had low resolution and nodal supports (tree no shown). All haplotypes of the P. plancyi complex, including $P$. p. fukienensis, were grouped together, in the same way as in the parsimony tree, with a posterior probability of 0.96 . However, the haplotypes of P. nigromaculatus did not form a clade, rather they formed a paraphyletic assemblage at the base of the tree.

Both POMC and TYR genes produced two well-defined clades: a $P$. nigromaculatus clade that includes all haplotypes of the $P$. nigromaculatus samples excluding CNU5268 and a P. plancyi clade that included all haplotypes of the $P$. plancyi complex excluding haplotypes of $P$. fukienensis. Both clades received moderate to high supports from both genes. The TYR gene tree had a lower resolution than the POMC gene tree; although the Bayesian analysis of $T Y R$ gene failed to group all haplotypes of $P$. nigromaculatus together (parsimony analysis did), members of the two clades were always well separated on every tree. A third group, the $P$. fukienensis clade that included all samples of $P$. fukienensis, was weakly supported by the $P O M C$ gene data, and not supported (neither contradicted) by the $T Y R$ gene data. Examining the sequence data directly, we found several fixed differences between the three clades. There were 2, 2, and 1 sites of the POMC gene and 2, 1, and 0 sites of the TYR gene that are fixed for the $P$. nigromaculatus clade, the $P$. plancyi clade and the $P$. fukienensis clade, respectively.

One individual (NU5268) appeared to be an $\mathrm{F}_{1}$ hybrid between $P$. plancyi and P. nigromaculatus. Two haplotypes from this individual were obtained by cloning and were grouped with the $P$. plancyi clade and the $P$. nigromaculatus clade, respectively. Directly examining the sequences, we found that CNU5268 was heterozygous at all polymorphic sites between $P$. plancyi and $P$. nigromaculatus. For example, at position 90 of POMC gene, all $P$. plancyi samples had a "C" while all $P$. nigromaculatus samples had a "T". CNU5268 was heterozygous on the site with " $\mathrm{C}$ " and "T". However, we cannot eliminate the possibility that this individual resulted from a recent backcross, e.g. $\mathrm{F}_{2}$ or $\mathrm{F}_{3}$, because we only examined two gene loci. 


\section{Mitochondrial cytochrome b gene (Cyt-b) genealogy}

Sequence data were collected from one mitochondrial gene, $C y t-b$. A total of $335 C y t-b$ sequences were gathered for the $P$. plancyi complex, including 333 new sequences from this study and two sequences from GenBank. A total of $330 C y t-b$ sequences were gathered for P. nigromaculatus, including 57 new sequences from this study and 273 sequences from several published sources. In addition, four sequences for three outgroup taxa, $P$. saharicus, $P$. lessonae, and P. porosus were obtained from GenBank. Sequences from different studies had different lengths; approximately half of the sequences included 1,043 nucleotide sites (full length) while others has 670850 sites at various positions. To accommodate the majority, a data set of 1043 sites was created. A total of 380 haplotypes were identified, including three for the outgroup taxa, and 393 sites were variable and 269 were phylogenetically informative. A GTR $+\mathrm{I}+\mathrm{G}$ model was selected as the best-fit model by the hierarchical likelihood ratio test. A Bayesian tree of $C y t-b$ along with the posterior probabilities is provided in Figure 4.

The Bayesian tree revealed several interesting features (Figure 4). Similar to the POMC gene trees (Figure 2), it resolved a $P$. fukienensis clade (clade A) at the very base of the tree. The member composition of the clade was exactly the same as the POMC tree. One outgroup taxon, $P$. porosus, was placed between the P. fukienensis clade and the other ingroup members. Unlike the nuclear gene trees, haplotypes of $P$. nigromaculatus and $P$. plancyi did not form two monophyletic groups, rather, they were intermingled and together formed one monophyletic group. Within this plancyi-nigromaculatus group, a Korean P. nigromaculatus clade (clade B) branched off first, which included all the $P$. nigromaculatus samples from the Korean Peninsula and adjacent locations of China (sites 2 and 3 and surrounding area; Figure 1). The sistergroup of the Korean $P$. nigromaculatus clade included a paraphyletic assemblage (group $\mathrm{C}$ ) of haplotypes of $P$. plancyi from central China (sites 5, 6, 7, 8, 9, $10,12,14,15,16,22,23,24$ and 25 but predominantly sites 6, 7, 9, 15, and 16; Figure 1) at the base and a wellsupported monophyletic group (clade D: the introgression clade) nested inside the paraphyletic group C. Clade $\mathrm{D}$ was the most interesting group and included the remaining $P$. nigromaculatus and $P$. plancyi haplotypes. Within this clade, again, haplotypes from $P$. nigromaculatus and P. plancyi failed to form respective clades; haplotypes of $P$. plancyi sporadically nested within "bushes" of $P$. nigromaculatus at 20 different places, except at the top of the tree where a large number of haplotypes of $P$. plancyi formed a clade (D3). The basal branches of clade D comprised haplotypes of $P$. nigromaculatus. Two haplotypes of $P$. nigromaculatus from western Sichuan and five haplotypes from Japan formed two well-supported groups (D1 and D2) at the very basal position of clade D. Although the overall nodal supports within clade D were low, some close associations between haplotypes of $P$. nigromaculatus and P. plancyi were well supported. Eight clades, where haplotypes from both species were found, received BPPs greater than 0.90 (Figure 4). Within each of these eight clades, haplotypes of $P$. nigromaculatus and $P$. plancyi were found to be identical or only different by one or two base pairs. In addition, the overall clado-pattern of the haplotypes within clade D bore little association with their geographic locations, except a few well-supported sister groups in which both members came from the same area.

The parsimony analysis found more than 400,000 MPTs with 879 steps $(C I=0.3936, R I=0.8733)$. Similar to the Bayesian tree, the MP tree also defined a P. fukienensis clade, clade $(\mathrm{B}+\mathrm{C}+\mathrm{D})$, clade $\mathrm{B}$, clade $(\mathrm{C}+\mathrm{D})$, a paraphyletic group $C$, and several other nodes within clade $\mathrm{D}$. Several close associations between $P$. nigromaculatus and $P$. plancyi within clade D were also identified. However, the other relationships among the haplotypes were poorly resolved compared to the Bayesian tree.

The estimated times of divergences between the major mitochondrial lineages were mapped on the $C y t-b$ gene tree (Figure 4). In general, these estimates were largely congruent with several other independent studies. For example, Lymberakis et al. estimated that the European and Far Eastern lineages were separated 15 MYA [21], and Sumida et al.'s estimate for this dichotomy was 5.910.9 MYA based on allozyme data [22]. These results were relatively close to our estimate of 13.40 MYA (CI 8.33-18.92). Sumida et al. estimated that the split of $P$. nigromaculatus and $P$. porosus took place at least 3 MYA [23], and it was again congruent with our estimate of 2.93 to 6.25 MYA for this event.

There were major differences between the nuclear and mitochondrial gene trees. Both nuclear genes resolved both $P$. plancyi and $P$. nigromaculatus as monophyletic clades, but the mitochondrial gene tree mixed haplotypes from the two species together. On the other hand, a major agreement among the nuclear $(P O M C)$ and mitochondrial gene trees was that all of them resolved a separated P. fukienensis clade. All new sequences are deposited in GenBank (accession numbers: Cyt-b GU977277GU977669; POMC GU977670-GU978222; TYR GU978223-GU978775). Aligned data sets that used to generate the phylogenetic hypotheses are presented as additional files 1,2 and 3.

\section{Discussion}

The multiple mitochondrial genome introgression hypothesis

The haplotypes of $P$. nigromaculatus and P. plancyi intertwined together on the mitochondrial gene tree (Figure 


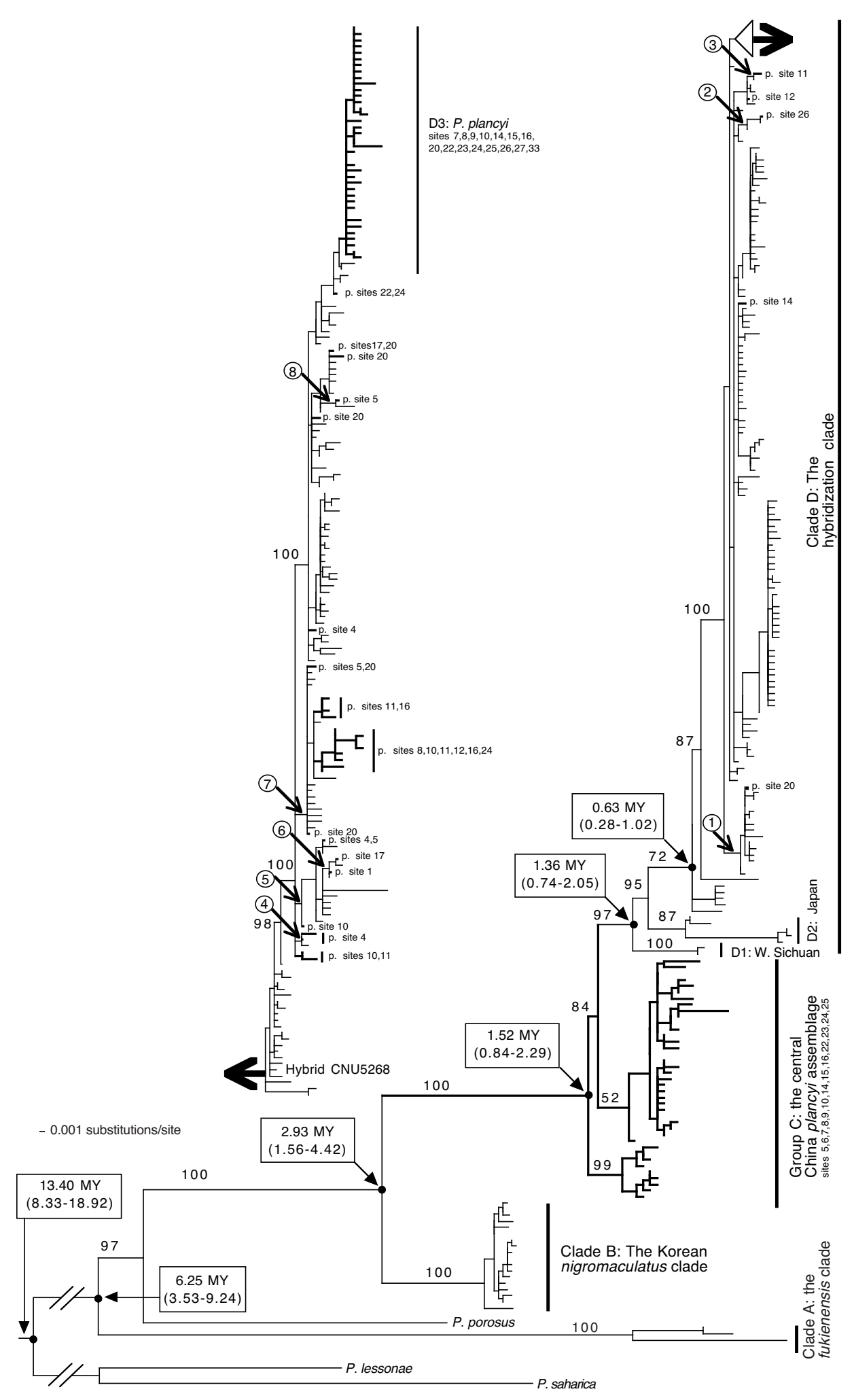

Figure 4 A Bayesian tree from the mitochondrial cytochrome $\boldsymbol{b}$ gene data. Bold branches and p. represent haplotypes of Pelophylax plancyi and others are $P$. nigromaculatus unless otherwise indicated. The numbers above the branches are Bayesian posterior probabilities. Divergence times were estimated from a reduced data set; divergence times in parentheses are confidence intervals at $95 \%$ level. Numbers within circles indicated close association between haplotypes of P. nigromaculatus and P. plancyi, which likely represent independent mitochondrial genome introgression events. 
4), despite the clear separation between the two species on both nuclear gene trees (Figure 2 and Figure 3). Other evidence, such as morphological and ecological traits, also strongly supports the validity of the two species [18]. The conflicting patterns between the nuclear and mitochondrial gene trees support Kim et al.'s hypothesis [16] that there were historical mitochondrial introgressions between $P$. nigromaculatus and $P$. plancyi. To best explain our data, we further propose a multiple mitochondrial genome introgression hypothesis: one ancient "forward" mitochondrial introgression event from $P$. plancyi to $P$. nigromaculatus followed by rampant recent "backward" introgression events from $P$. nigromaculatus to $P$. plancyi.

Our nuclear gene data conform to several previous studies and suggest that the P. plancyi and P. nigromaculatus are sistergroups $[18,21]$. The Korean $P$. nigromaculatus clade (clade B) on the Cyt-b gene tree likely represents the only "true" mitochondrial lineage of $P$. nigromaculatus, and its sistergroup (clade $\mathrm{C}+\mathrm{D}$ ) likely represents the mitochondrial lineage of P. plancyi (Figure 4). The paraphyletic group C (the central China P. plancyi assemblage) likely represents the "original" mitochondrial lineages of $P$. plancyi, which have always been associated with $P$. plancyi nuclear genomes. The introgression clade $\mathrm{D}$, which nests within group $\mathrm{C}$, represents an introgressed $P$. plancyi mitochondrial lineage (Figure 4). Within this clade, $P$. plancyi individuals are sporadically dispersed throughout the "bushes" of $P$. nigromaculatus, and the four basal sub-clades comprise $P$. nigromaculatus individuals exclusively. The most parsimonious explanation for this pattern is that all individuals in this clade share a common mitochondrial ancestor derived from an ancient hybridization event between a female $P$. plancyi and a male $P$. nigromaculatus. The introgressed $P$. plancyi genome subsequently expanded to the majority of the distribution range of $P$. nigromaculatus except the Korean Peninsula and its adjacent areas. The nested $P$. plancyi lineages represent $P$. plancyi individuals, which "took back" their mitochondrial genomes from P. nigromaculatus via recent hybridization events. Of the 20 nested placements of $P$. plancyi, there are cases where the closest $P$. nigromaculatus relatives are not identified due to the unresolved relationships on the tree. Nevertheless, there are eight well-supported sub-clades $(\mathrm{BPP}>0.90)$ that embrace both $P$. plancyi and $P$. nigromaculatus individuals. Each of these sub-clades likely represents a recent mitochondrial introgression event from $P$. nigromaculatus to $P$. plancyi. The introgressions between the two species appear to be bi-directional and, at present, the majority of both species possess introgressed, rather than "original", mitochondrial genomes.

Overall, the multiple mitochondrial genome introgression hypothesis most parsimoniously accounts for our data and also immediately provides an explanation for the rampant introgression within the introgression clade (clade D). In this clade, all $P$. nigromaculatus individuals do, in fact, possess historical mitochondrial genomes of $P$. plancyi, and the mitochondrial introgression from $P$. nigromaculatus to $P$. plancyi actually results in re-union of the mitochondrial and nuclear genomes of $P$. plancyi. The two genomes are already co-adapted, and therefore, the introgression might have little negative impact on the fitness of the hybrids.

A re-examination of the mitochondrial gene tree of the two species, by increasing the number of taxa or number of informative characters (nucleotide sites), may change the topology and favor a different explanation. However, our sampling of taxa (populations) is robust and more taxa are unlikely to change the topology. More data could improve the resolution of the tree, particularly within clade D. Those will unlikely change the essentials of the current hypothesis but will likely help to resolve the number of recent introgression events within clade D. The intertwined pattern of the mitochondrial gene tree may also be explained by incomplete lineage sorting [12], however, it is probably not valid in our case. Due to the maternal mode of inheritance, mitochondrial genes are expected to reach coalescence up to four times faster than nuclear genes [24]. In our study, both nuclear genes (particularly $P O M C$ ) have reached coalescence at the species level, and it is therefore unlikely that the more rapidly evolving mitochondrial genes have not.

\section{Diversification and introgression of the $P$. nigromaculatus- plancyi mitochondrial genomes in time and space}

The Korean nigromaculatus clade (clade B), which includes all samples from the Korean Peninsula and adjacent locations of China, was separated from the rest at approximately 2.93 MYA (Figure 4). Since the Korean nigromaculatus clade represents the only "true" $P$. nigromaculatus mitochondrial lineage while all other $P$. nigromaculatus and $P$. plancyi possess a $P$. plancyi mitochondrial genome, this split may coincide with the speciation event that separated $P$. nigromaculatus and $P$. plancyi. All other $P$. nigromaculatus possess an introgressed $P$. plancyi mitochondrial genome. This "true" $P$. nigromaculatus mitochondrial lineage might have survived in the Korean Peninsula refugium during ice ages and expanded to nearby areas of China during the interglaciation periods (sites 2 and 3, Figure 1). The Korean Peninsula is a well-recognized refugium during several glaciation cycles and has provided shelters for many ancient lineages $[25,26]$. A recent phylogeographic study of $P$. nigromaculatus by Zhang et al. also identified two major clades, a Korean clade including samples from the Korean Peninsula and adjacent China and a main clade including the Japanese and most Chinese samples [27]. Without realizing that the main clade of $P$. nigromacula- 
tus was actually descended from P. plancyi, they hypothesized that the Gunz glaciation (0.9-1.2 MYA) might be the cause of allopatric isolation and lineage splitting. Our divergence time estimate (2.93 MYA) is older than that of Zhang et al.'s [27], and the observed divergence is not a lineage split within P. nigromaculatus, rather, it represents the separation between the two species.

The central China P. plancyi mitochondrial lineages share a most recent common ancestor with clade D at approximately 1.52 MYA (Figure 4). The central China lineages may represent the only survivors of the "original" P. plancyi mitochondrial genome. The majority members of these lineages are from sites 6, 7, 9, 15 and 16 in the vicinity of Da-Bie-Shan Mountains (site 9; Figure 1). The persistence of these ancient lineages may be attributed to the Da-Bie-Shan refugium. The Da-Bie-Shan and surrounding area is hypothesized to have been a glaciation refugium since the Tertiary and maintains many ancient endemic species $[28,29]$. These $P$. plancyi mitochondrial lineages may have survived in the refugium and expanded to the surrounding area (sites $5,6,7,8,9,10,12,14,15$, 16, 22, 23, 24, and 25; Figure 1).

Clade D represents an ancient mitochondrial genome introgression event that occurred at least 1.36 MYA (CI 0.74-2.05). Most previous reported cases of mitochondrial introgression are recent and the introgressed mitochondrial genomes in the recipient species are identical or nearly so to those in the donor species $[2,6]$. The two reported exceptions are crotaphytid lizards and Scutiger frogs. McGuire et al. reported repeated introgression events between Crotaphytus collaris and C. bicinctores and some could be as old as 2.5 MYA [4]. Chen et al. reported a case between two species of Scutiger, which was dated at approximately 3.5-9.5 MYA [8]. With increasing haplotype divergence, it becomes more difficult to discriminate between introgression and incomplete lineage sorting [30], but our data provide a convincing case of ancient mitochondrial genome introgression. We could not locate where this introgression event might have occurred from our data; repeated glaciation circles and associated range expansions/contractions may have rendered it difficult, if not impossible.

Within the introgression clade (D), multiple introgression events have taken place over time and space. There are at least eight well-supported sub-clades that demonstrate close associations between $P$. nigromaculatus and $P$. plancyi and represent at least eight independent introgression events. If we consider those sub-clades that did not receive high nodal supports, introgressions from $P$. nigromaculatus to $P$. plancyi may have independently taken place as many as 20 times. All these introgression events are probably recent. The common ancestor of the clade that includes most of clade D excluding D1 and D2, is 0.63 MYA (CI 0.28-1.02); therefore, all divergence within this clade should be younger than this time estimate. Within these eight well-supported sub-clades, the differences between $P$. nigromaculatus and $P$. plancyi are small: some are identical while the largest differences are two of the 670 base pairs that were compared. Furthermore, the introgression events may have occurred at various locations. Within the eight well-supported subclades, closely associated haplotypes of $P$. nigromaculatus and $P$. plancyi are often from the same area. For example, all individuals of both species in sub-clade 2 are from Zhang Jia Jie (site 26), and in sub-clade 3, all individuals are from Zhejiang Province of China (sites 11 and 13). Interestingly, the Korean P. plancyi is not closely related to the Korean $P$. nigromaculatus, suggesting that the Korean $P$. plancyi may not be indigenous, and is probably recently established. In addition, the hybridization between the two species appears to be an ongoing process and this is evidenced by an F1 hybrid identified in the present study.

\section{Mechanisms of mitochondrial genome introgression and replacement}

Asymmetrical reproductive ability of hybrids and continuous backcrossing are likely responsible for the observed mitochondrial introgression and replacement between the two green pond frogs. Kawamura and Nishioka reported that all male $\mathrm{F}_{1}$ hybrids between $P$. nigromaculatus and P. plancyi chosenicus were sterile, but half of the female $F_{1}$ hybrids were probably fertile [31]. Both field observation and lab experiments also confirmed that among crosses between P. nigromaculatus and P. porosus, females were partially fertile but males were completely sterile [32]. Haldane's rule predicts that when in $\mathrm{F}_{1}$ offspring of two animal species, one sex is sterile, the sex is usually the heterogametic sex. Pelophylax nigromaculatus has an XX/XY sex determination [33], and therefore, the above observations are consistent with Haldane's rule. While frequent inter-specific hybridization is the necessary first step toward mitochondrial genome introgression, such asymmetrical reproductive ability of hybrids would greatly facilitate backcrossing and the disappearance of hybridization signals in the nuclear genome while the introgressed mitochondrial genomes remain intact. Other mechanisms, such as asymmetries in species abundance or mating preferences and male-biased colonization [2,34] may also play a role in promoting mitochondrial introgression between the two green pond frogs.

The introgressed mitochondrial genomes in both $P$. nigromaculatus and $P$. plancyi expanded to the majority of their distribution ranges. McGuire et al. proposed two non-exclusive explanations for mitochondrial replacement across an extended geographic area: frequent hybridization events followed by genetic drift, or selective 
sweeps associated with rare hybridization [4]. The introgression from $P$. nigromaculatus back to $P$. plancyi is clearly a case of multiple rampant introgression events, and hence is consistent with McGuire et al's hypothesis [4]. On the other hand, the introgression from P. plancyi to $P$. nigromaculatus (the ancient introgression event) appeared to have occurred only once, so selective sweep might have been involved but we do not have data for this hypothesis. Thermal adaptation has been proposed for selection in several cases [7]. Nevertheless, no direct evaluation of the selective advantage of introgression event has so far been conducted. Theoretically, when species invade a new area, capturing an already adapted mitochondrial genome from the local species may provide the invader with a selective advantage [12].

\section{Taxonomic implications}

Considering information from all three genes, Pelophylax fukienensis is a valid species. The nuclear gene POMC indicates that all its samples form a monophyletic group and possess fixed differences at several nucleotide sites from P. plancyi. At site 17, P. fukienensis, P. nigromaculatus and $P$. plancyi are sympatric. Of the 22 specimens that we examined, no hybrid was found, suggesting established reproductive isolation between the three species. Furthermore, the P. fukienensis clade is separated from all other $P$. plancyi by another valid species, $P$. porosus, on the mitochondrial gene tree. Pope first named the species, "Rana" fukienensis [35], but most recent authors considered it as a synonym of $P$. plancyi [19]. Based on molecular data, Sumida et al. recently suggested that it is a valid species, and is likely more closely related to $P$. porosus than to $P$. nigromaculatus [22]. Its distribution may include the island of Taiwan and the adjacent coast region of mainland China. However, many populations from inland of China, which were previously diagnosed as $P$. fukienensis (e.g. sites 10,11, 12, 16 in [18]), are in fact P. plancyi.

Our data do not support the validity of $P$. hubeiensis and $P$. chosenicus. Both mitochondrial and nuclear genes failed to reveal any distinctiveness of the populations under the two names. The Korean populations of P. plancyi $(=P$. chosenicus $)$ may have only invaded the Peninsula recently. Their reported morphological differences [18] are likely geographic intra-specific variations.

\section{Conclusions}

There are multiple mitochondrial introgression events between the two green pond frog species, including one ancient "forward" introgression event from $P$. plancyi to $P$. nigromaculatus followed by rampant recent "backward" introgression events from $P$. nigromaculatus to $P$. plancyi.
The majority of individuals in the two species have either introgressed (P. nigromaculatus) or reclaimed (P. plancyi) mitochondrial genomes while no trace of past hybridization in their nuclear genomes was detected. The mitochondrial introgression between these two species is unique in several ways. First, one introgression event is ancient, estimated at 1.36 MYA. Most previous reported cases of mitochondrial introgression are recent $[2,6]$. Second, independent introgression events from $P$. nigromaculatus to $P$. plancyi result in re-constitution of cytonuclear association of $P$. plancyi. This is the first such case. Third, there are multiple (as many as 20) but spatially and temporally separated introgression events. Such introgression is likely an ongoing process; the $F_{1}$ hybrid that we found suggests that hybridization between the two species may frequently occur.

Mitochondrial genome introgression and replacement may be more common than we previously perceived. For example, inter-specific hybridization among amphibians is common, and mechanism that can produce mitochondrial introgression, such as asymmetrical reproductive ability of hybrids, is also commonly observed ([36,37]; Additional examples in Duellman and Trueb [38]). The common occurrence of such hybridization events likely has produced numerous mitochondrial genome introgressions in nature populations waiting to be detected.

Using mitochondrial genes alone to infer species history or to determine species status can be misleading, and mtDNA only represents an incomplete history of a species [12]. This message has been repeatedly demonstrated by numerous studies. A large number of mtDNA based phylogeographic studies and their conclusions may need to be revisited.

This mitochondrial introgression between $P$. nigromaculatus and P. plancyi provides a unique opportunity to study cyto-nuclear interaction and co-adaptation. Within $P$. nigromaculatus and $P$. plancyi, there are nuclear genomes interacting with their "original" and "coevolved" mitochondrial genomes, and those interacting with "introgressed" foreign mitochondrial genomes. If the two genomes in every species were co-adapted, a recent mitochondrial introgression would most likely create mismatches between the mitochondrial vs. nuclearencoded components (e.g. subunits for the electron transport chains) and cause functional disruption [15]. Such matches or mismatches can be examined and compared for different populations. Recent development in proteomics have provided us powerful tools to model the protein structure [39] and investigate the potential impact of these mismatches on the function of the protein molecules, which in turn may have significant impacts on the fitness of the hybrid lineages. 


\section{Methods Sampling}

An extensive coverage, particularly of the mitochondrial lineages of the two species, is essential for investigating the extent of mitochondrial introgression. A total of 333 specimens of the Pelophylax plancyi complex from 23 collecting sites were examined in this study, which included all four subspecies (plancyi, fukienensis, hubeiensis, and chosenicus), and covered most of the species' distribution (Figure 1). In addition, mitochondrial $C y t-b$ gene sequences of one sample from the Korean Peninsula (AF205087) and one from the island of Taiwan (AB029941) were obtained from GenBank.

A total of 60 specimens of $P$. nigromaculatus from 29 collecting sites were included in this study, which covered the majority of the species' distribution and represented the three main lineages defined by a previous study [27]. In addition, 273 mitochondrial $C y t-b$ gene sequences representing samples from 75 locations were also obtained from GenBank (DQ006233-DQ006267, AY803813AY803895, AJ880539-AJ880677, $\underline{\text { AY355755-AY355757, }}$ AF205087, AF274929, AF467981, AB029937, AB036396, AB043889, NC002805) and other published sources. Most of the sequences were from four previous studies of the species $[16,23,27,40]$. We did not sequence many new individuals of $P$. nigromaculatus because a large number of sequences were already available. Four nuclear gene sequences (POMC AB360151; TYR D12514, AY322363. DQ360045) were also obtained from GenBank.

Eight ranid species, Pelophylax lessonae, $P$. saharicus, $P$. perezi, P. porosus, Babina pleuraden, Rana shuchinae, Staurois latopalmatus, Lithobates catesbeianus, were selected as outgroup taxa. Most of these species were considered closely related to $P$. plancy $i$ and $P$. nigromaculatus $[18,20,21,41]$. All their sequences were obtained from GenBank (POMC AY819106, AB360150; TYR DQ360042, DQ360057, AY322347; Cyt-b DQ474177, EU047779, AB029938, AB036402). Different dataset used different outgroup combinations depending on the availability of data. Detailed information of specimens and collecting sites are listed in Additional file 1 and shown in Figure 1.

\section{Laboratory protocols}

We sequenced one fragment from the mitochondrial genome and two fragments from the nuclear genome. The mitochondrial $C y t-b$ gene is one of most frequently used genes in vertebrates for phylogenetic construction, and several previous studies of $P$. nigromaculatus used this gene fragment $[27,40]$. By using the same fragment, we were able to incorporate the published data in our analysis. From the nuclear genome, we selected the $P O M C$ and TYR as markers because they have been extensively used for ranid species and both demonstrate variability at population level. All primers used in this study are listed in Table 1.

Genomic DNA was isolated from liver or muscle tissues using a standard phenol/chloroform extraction protocol [42]. Standard polymerase chain reaction (PCR) amplification was performed with an annealing temperature that was optimized for each primer pair (Table 1). All PCR products were verified on $1 \%$ agarose gels and purified using QIAquick PCR purification kits (Qiagen). The purified products were directly cycle-sequenced with the same primers from both directions. All DNA sequencing reactions were performed using BigDye terminator sequencing chemistry with an ABI 3730 (Applied Biosystems) automatic sequencer.

Nuclear fragments that contained overlapping peaks (double nucleotide calls), were cloned using a pGEM-T Easy Vector System I (Promega) to verify the sequence of each haplotype in heterozygous individuals. Restriction enzyme reaction (EcoRI blue/white cloning qualified EcoRI restriction enzyme, Promega) was first performed using plasmid extraction in white clones to determine the colonies that contain positive inserts. Bacterial-PCR was then performed using selected positive individual clones as templates. The PCR products were purified and sequenced following the same protocols specified above.

\section{Data analysis}

All sequences were checked and edited using BioEdit (version 7) [43]. All alignments were completed using MacClade (version 4) [44]. All three fragments were from coding regions and therefore the alignment was straightforward. Prior to phylogenetic analysis of the haplotypes, recombination tests were conducted for all nuclear gene sequences. If recombination occurs within a fragment, phylogenetic methods that produce cladograms (bifurcating trees) would be inappropriate.

We used Sawyer's [45] method to test for recombination. Following the author's recommendation, the default parameters of the computer program Geneconv (Version 1.81) [46] were used, which include the highest acceptable $P$ value of 0.05 , Bonferroni correction for multiple comparisons, scanning sequence pairs, and a permutation of 10,000 . The mismatch penalties parameter was varied from small (gscale $=1)$ to infinite $($ gscale $=0)$ to allow sequence mismatch within each potentially conversed gene fragment.

A phylogenetic analysis was conducted to establish the genealogy of the DNA haplotypes. Both maximum parsimony method and Bayesian inference were used. The parsimony analysis was conducted with PAUP* (version 4.0b10) [47]. All characters were equally weighted and unordered. All phylogenetically uninformative characters were excluded from analysis. Heuristic searches with 1000 random sequence addition replicates were used 
Table 1: Primers and annealing temperatures used for PCR and sequencing in this study

\begin{tabular}{cllcc}
\hline Gene & Primer & Primer Sequences (5'-3') & Annealing T & Reference \\
\hline Cyt- $b$ & B104(F) & AAC ATC TCT GCA TGA TGA AAC TTC GG & $55^{\circ} \mathrm{C}$ & This study \\
& B829(R) & AT TGA GCG AAG GAT GGC GTA GGC GAA & & Wiens et al. [56] \\
POMC & POMC-1(F) & GAA TGT ATY AAA GMM TGC AAG ATG GWC C & $50^{\circ} \mathrm{C}$ & \\
& POMC-2(R) & TAY TGR CCC TTY TTG TGG GCR TT & $50^{\circ} \mathrm{C}$ & Bossuyt and Milinkovitch [57] \\
& POMC-3(F) & TCT GCM GAR TCW CCY GTG TTT CC & $50^{\circ} \mathrm{C}$ & \\
\hline \multirow{2}{*}{ TYR } & POMC-4(R) & TGG CAT TYT TGA AAA GAG TCA T & & \\
& TyrlB (F) & AGG TCC TCY TRA GGA AGG AAT G & & \\
\hline
\end{tabular}

with tree bisection reconnection (TBR) branch swapping. Due to the large number of similar haplotypes, a $100,000,000$ rearrangement limit was imposed on each replicate to reduce the computation time. Nodal support was estimated with bootstrap analyses [48] using 100 replicates. Within each bootstrap replicate, 10 random sequence addition replicates were conducted with a 100,000,000 rearrangement limit on each replicate.

The Bayesian analysis was conducted with MrBayes (version 3.2) [49]. A best-fit DNA substitution model was first selected by MrModeltest (version 2.1) [50]. A flat "prior" setting was used in MrBayes and four Markov chains were executed. Each dataset was run for $10,000,000$ generations and trees were sampled every 500 generations. We used the last 10,000 sampled trees to estimate the consensus tree and the Bayesian posterior probabilities, and all other trees were designated as "burn-in". Tracer (version 1.4) [51] was used to plot the resulting likelihood values and to determine when the Markov chains reached convergence. Two separate runs, which included a total of four independent tree searches, were conducted and the resulting trees were compared and pooled.

For divergence time estimates, a Bayesian method with computer program BEAST (version 1.5.1) [52] was used. We were only interested in the divergence times of the major clades, therefore, a simplified data set was constructed for the BEAST analysis. Most similar haplotypes of $P$. fukienensis, P. nigromaculatus and P. plancyi were excluded, and only eleven haplotypes were included, which represented the major lineages. Nine additional taxa, Glandirana rugosa (AF205093), Pelophylax bedriagae (DQ474141), P. cretensis (DQ474147), P. epeirotica (DQ474153), P. kurtmuelleri (DQ474156), P. lessonae (EU047779), P. perezi (DQ902146), P. porosus (AB029938, AB036402), and P. saharicus (DQ474177) were introduced to provide a calibration point and a root for the tree. We used the separation of the island of Crete from the mainland as a calibration point, which corresponds to the separation of $P$. cretensis from the common ancestor of P. bedriagae, P. epeirotica and P. kurtmuelleri [21]. Geological studies dated the separation at 5-5.5 MYA $[53,54]$. A HKY $+\mathrm{I}+\mathrm{G}$ was used to describe the substitution model, a Yule process was used to describe speciation and an uncorrelated lognormal (UCLN) model was used to describe the relaxed clock [55]. BEAST was run for $80,000,000$ generations with samples taken every 1,000. Three independent MCMC runs were conducted.

\section{Additional material}

Additional file 1 Aligned cytochrome $b$ sequence data for Pelophylax
fukienensis, P. nigromaculatus and P. plancyi.
Additional file 2 Aligned POMC sequence data for Pelophylax fukien-
ensis, P. nigromaculatus and P. plancyi.
Additional file 3 Aligned tyrosinase sequence data for Pelophylax fuk-
ienensis, P. nigromaculatus and P. plancyi.
Additional file 4 Specimen and sampling site information for Pelo-
phylax fukienensis, P. nigromaculatus and P. plancyi.

Authors' contributions

All authors participated in the project design and sample collecting. $\mathrm{KL}$ and FW carried out most of the fieldwork and molecular data collection, and participated in data analysis. WC, LT and MM commented on manuscript drafts. KB participated in data collecting and analysis. JF conceived the project, participated in data analysis and finalized the manuscript. All authors read and approved the final manuscript.

\section{Acknowledgements}

We would like to thank S. Huang, N. Poyarkov, F. Zhang and Y. Zheng for their laboratory and field assistance. We also thank J. Bogart for his valuable comments on an early version of this manuscript. This project is supported by a CNU grant to LT and WC, and a NSERC discovery grant to JF.

\section{Author Details}

${ }^{1}$ College of Life Sciences, Capital Normal University, Beijing 100048, China, ${ }^{2}$ College of Veterinary Medicine, Seoul National University, Seoul 151-742, South Korea and ${ }^{3}$ Department of Integrative Biology, University of Guelph, Guelph, Ontario N1G 2W1, Canada

Received: 9 December 2009 Accepted: 29 June 2010 Published: 29 June 2010 


\section{References}

1. Wilson CC, Bernatchez $\mathrm{L}$ : The ghost of hybrids past: fixation of arctic charr (Salvelinus alpinus) mitochondrial DNA in an introgressed population of lake trout (S. namaycush). Mol Ecol 1998, 7:127-132.

2. Melo-Ferreira J, Boursot P, Suchentrunk F, Ferrand N, Alves PC: Invasion from the cold past: extensive introgression of mountain hare (Lepus timidus) mitochondrial DNA into three other hare species in northern Iberia. Mol Ecol 2005, 14:2459-2464.

3. Melo-Ferreira J, Boursot P, Randi E, Kryukov A, Suchentrunk F, Ferrand N, Alves PC: The rise and fall of the mountain hare ((Lepus timidus) during Pleistocene glaciations: expansion and retreat with hybridization in the Iberian Peninsula. Mol Ecol 2007, 16:605-618.

4. McGuire JA, Linkem CW, Koo MS, Hutchison DW, Lappin AK, Orange DI, Lemos-Espinal J, Riddle BR, Jaeger JR: Mitochondrial introgression and incomplete lineage sorting through space and time: phylogenetics of crotaphytid lizards. Evolution 2007, 61:2879-2897.

5. Sota T: Radiation and reticulation: extensive introgressive hybridization in the carabid beetles Ohomopterus inferred from mitochondrial gene genealogy. Popul Ecol 2002, 44:145-156

6. Ballard JWO: When one is not enough: introgression of mitochondrial DNA in Drosophila. Mol Biol Evol 2000, 17:1126-1130.

7. Bernatchez L, Glémet H, Wilson CC, Danzmann RG: Introgression and fixation of Arctic char (Salvelinus alpinus) mitochondrial genome in an allopatric population of brook trout (Salvelinus fontinalis. Can J Fish Aquat Sci 1995, 52:179-185.

8. Chen W, Bi K, Fu J: Frequent mitochondrial gene introgression among high elevation Tibetan megophryid frogs revealed by conflicting gene genealogies. Mol Ecol 2009, 18:2856-2876.

9. Abernethy K: The establishment of a hybrid zone between red and sika deer (genus Cervus. Mol Ecol 1994, 3:551-562.

10. Roca AL, Georgiadis N, O'Brien SJ: Cytonuclear genomic dissociation in African elephant species. Nat Genet 2005, 37:96-100.

11. Ruedi M, Smith MF, Patton JL: Phylogenetic evidence of mitochondrial DNA introgression among pocket gophers in New Mexico (family Geomyidae). Mol Ecol 1997, 6:453-462.

12. Ballard JWO, Whitlock MC: The incomplete natural history of mitochondria. Mol Ecol 2004, 13:729-744.

13. Avise JC: Phylogeography, the History and Formation of Species. Cambridge Harvard University Press; 2000.

14. Beheregaray LB: Twenty years of phylogeography: the state of the field and the challenges for the southern hemisphere. Mol Ecol 2008, 17:3754-3774

15. Ellison CK, Burton RS: Disruption of mitochondrial function in interpopulation hybrids of Tigriopus californicus. Evolution 2006, 60:1382-1391

16. Kim JB, Matsui M, Lee JE, Min MS, Suh JH, Yang SY: Notes on a discrepancy in mitochondrial DNA and allozyme differentiation in a pond frog Rana nigromaculata. Zool Sci 2004, 21:39-42.

17. Spolsky C, Uzzell T: Natural interspecies transfer of mitochondrial DNA in amphibians. Proc Natl Acad Sci USA 1984, 81:5802-5805.

18. Fei L, Ye C, Jiang J, Xie F, Huang Y: An Illustrated Key to Chinese Amphibians. Chengdu, Sichuan Publishing House of Science and Technology; 2005. [in Chinese]

19. Zhao E, Adler K: Herpetology of China. Oxford, SSAR; 1993.

20. Frost DR: Amphibian Species of the World: an Online Reference. [http:/ /research.amnh.org/herpetology/amphibia//. American Museum of Natural History, New York, USA Version 5.3 (12 February, 2009). Electronic Database

21. Lymberakis P, Poulakakis N, Manthalou G, Tsigenopoulos CS, Magoulas A, Mylonas M: Mitochondrial phylogeography of Rana (Pelophylax) populations in the Eastern Mediterranean region. Mol Phylogenet Evol 2007, 44:115-125

22. Sumida M, Ogata M, Nishioka M: Molecular phylogenetic relationships of pond frogs distributed in the Palearctic region inferred from DNA sequences of mitochondrial $12 \mathrm{~S}$ ribosomal RNA and cytochrome $b$ genes. Mol Phylogenet Evol 2000, 16:278-285

23. Sumida M, Ogata M, Kaneda H, Yonekawa H: Evolutionary relationships among Japanese pond frogs inferred from mitochondrial DNA sequences of cytochrome $b$ and 125 ribosomal RNA genes. Genes Genet Syst 1998, 73:121-133.
24. Palumbi SR, Cipriano F, Hare MP: Predicting nuclear gene coalescence from mitochondrial data: the three-times rule. Evolution 2001, 55:859-868

25. Chung MG, Kang SS: Genetic variation and population structure in Korean populations of Eurya japonica (THEACEAE). Am J Bot 1994 81:1077-1082.

26. Serizawa K, Suzuki H, Iwasa MA, Tsuchiya K, Pavlenko MV, Kartavtseva IV, Chelomina GN, Dokuchaev NE, Han S-H: A spatial aspect on mitochondrial DNA genealogy in Apodemus peninsulae from east Asia Biochem Geneti 2002, 40:149-161.

27. Zhang H, Yan J, Zhang G, Zhou K: Phylogeography and demographic history of Chinese black-spotted frog populations (Pelophylax nigromaculata): evidence for independent refugia expansion and secondary contact. BMC Evol Biol 2008, 8:21.

28. Zhou Y, Feng Z: Study on flora of Mount Longwang, Zhejiang Province. $J$ East China Normal Univ (Nat Sci 1994:88-94. [in Chinese]

29. Chen Y, Zhou Z, Xu R, Shen J: A morphological examination of pollens in the Anhui Yaoluoping National Reserve and its ecological factors. Acta Micropal Sin 2008, 25:168-184. [in Chinese]

30. Funk DJ, Omland KE: Species-level paraphyly and polyphyly: frequency, causes, and consequences, with insights from animal mitochondrial DNA. Annu Rev Ecol Evol Syst 2003, 34:397-423.

31. Kawamura T, Nishioka M: On the pond frogs in the palearctic region, with special reference to the isolating mechanism between different species. Proc Japanese Soc Syst Zool 1975, 11:61-78.

32. Sumida M, Ishihara T: Natural hybridization and introgression between Rana nigromaculata and Rana porosa porosa in central Japan. Amphibia-Reptilia 1997, 18:249-257.

33. Li S: Cytotaxonomy of Amphibians in China. Beijing, Science Press; 2007. [In Chinese]

34. Thulin CG, Tegelström H: Biased geographical distribution of mitochondrial DNA that passed the species barrier from mountain hares to brown hares (genus Lepus): an effect of genetic incompatibility and mating behaviour. J Zool Lond 2002, 258:299-306.

35. Pope $\mathrm{CH}$ : Notes on amphibians from Fukien, Hainan, and other parts of China. Bull Am Mus Nat Hist 1931, 59:397-611.

36. Blair WF: Evolution in the Genus. Bufo. Austin, University of Texas Press; 1972

37. Vogel LS, Johnson SG: Estimation of hybridization and introgression frequency in toads (genus: Bufo) using DNA sequence variation at mitochondrial and nuclear loci. J Herpetol 2008, 42:61-75.

38. Duellman WE, Trueb L: Biology of Amphibians. Baltimore, The John Hopkins University Press; 1994

39. Zhang Y: Progress and challenges in protein structure prediction. Curr Opin Struc Biol 2008, 18:342-348.

40. Yang Y-H, Zhang D-X, Li Y-M, Ji Y-J: Mitochondrial DNA diversity and preliminary biogeographic inference of the evolutionary history of the black-spotted pond frog Rana nigromaculata populations in China. Acta Zool Sin 2004, 50:193-201. [in Chinese]

41. Che J, Pang J, Zhao H, Wu GF, Zhao EM, Zhang YP: Phylogeny of Raninae (Anura: Ranidae) inferred from mitochondrial and nuclear sequences. Mol Phylogenet Evol 2007, 43:1-13.

42. Palumbi SR: Nucleic acids II: The polymerase chain reaction. In Molecular Systematics 2nd edition. Edited by: Hillis DM, Moritz C, Mable BK. Sunderland, Sinauer Associates; 1996:205-247.

43. Hall TA: BioEdit: a user-friendly biological sequence alignment editor and analysis program for Windows 95/98/NT. Nucleic Acids Symp Ser 1998, 41:95-98.

44. Maddison DR, Maddison WP: MacClade 4. Analysis of phylogeny and character evolution. Sunderland, Sinauer Associates; 2003.

45. Sawyer S: Statistical tests for detecting gene conversion. Mol Biol Evol 1989, 6:526-538.

46. Sawyer S: GENECONV: Statistical Tests for Detecting Gene Conversion (Version 1.81). Washington University; 2000. Computer program distributed by the author

47. Swofford DL: PAUP*: Phylogenetic Analysis Using Parsimony (and Other Methods). Sunderland, Sinauer Associates; 2002.

48. Felsenstein J: Confidence limits on phylogenies: an approach using the bootstrap. Evolution 1985, 39:783-791.

49. Ronquist F, Huelsenbeck JP: MRBAYES 3: Bayesian phylogenetic inference under mixed models. Bioinformatics 2003, 19:1572-1574. 
50. Nylander JAA: MrModeltest version 2.1. Uppsala, Uppsala University; 2004. Computer program distributed by the author

51. Rambaut A, Drummond AJ: Tracer: MCMC Trace Analysis Package (version 1.4). 2007. Computer programs distributed by the authors

52. Drummond AJ, Rambaut A: BEAST: Bayesian evolutionary analysis by sampling trees. BMCEvol Biol 2007, 7:214.

53. Dermitzakis DM: Paleogeography, geodynamic processes and event stratigraphy during the Late Cenozoic of the Aegean area. Accademia Nazionale de. Lincei 1990, 85:263-288.

54. Beerli P, Hotz H, Uzzell T: Geologically dated sea barriers calibrate a protein clock for Aegean water frogs. Evolution 1996, 50:1676-1687.

55. Drummond AJ, Ho SY, Phillips MJ, Rambaut A: Relaxed phylogenetics and dating with confidence. PLOS Biol 2006, 4:e88.

56. Wiens JJ, Fetzner JW Jr, Parkinson CL, Reeder TW: Hylid frog phylogeny and sampling strategies for speciose clades. Syst Biol 2005, 54:778-807.

57. Bossuyt F, Milinkovitch MC: Convergent adaptive radiations in Madagascan and Asian ranid frogs reveal covariation between larval and adult traits. Proc Natl Acad Sci USA 2000, 97:6585-6590.

doi: $10.1186 / 1471-2148-10-201$

Cite this article as: Liu et al., Rampant historical mitochondrial genome introgression between two species of green pond frogs, Pelophylax nigromaculatus and P. plancyi BMC Evolutionary Biology 2010, 10:201

Submit your next manuscript to BioMed Central and take full advantage of:

- Convenient online submission

- Thorough peer review

- No space constraints or color figure charges

- Immediate publication on acceptance

- Inclusion in PubMed, CAS, Scopus and Google Scholar

- Research which is freely available for redistribution

Submit your manuscript at www.biomedcentral.com/submit
Ciomed Central 